Revue d'histoire de l'Amérique française

REVUE D.HISTOIRE DE L'AMÉRIQUE FRANÇAISE

\title{
H.-M. Caiserman et l'École littéraire de Montréal. Vers une exploration en yiddish du Canada français
}

\section{Pierre Anctil}

Volume 66, numéro 1, été 2012

URI : https://id.erudit.org/iderudit/1021082ar

DOI : https://doi.org/10.7202/1021082ar

Aller au sommaire du numéro

\section{Éditeur(s)}

Institut d'histoire de l'Amérique française

\section{ISSN}

0035-2357 (imprimé)

1492-1383 (numérique)

Découvrir la revue

\section{Citer cet article}

Anctil, P. (2012). H.-M. Caiserman et l'École littéraire de Montréal. Vers une exploration en yiddish du Canada français. Revue d'histoire de l'Amérique française, 66(1), 65-83. https://doi.org/10.7202/1021082ar

\section{Résumé de l'article}

$\mathrm{Au}$ début du $\mathrm{XX}^{\mathrm{e}}$ siècle, un certain nombre d'immigrants européens ont jeté un regard sur la société québécoise et ont rédigé à ce sujet, à Montréal, des textes littéraires en langue non officielle. C'est entre autres le cas d'un activiste culturel et leader communautaire d'origine roumaine, Hananiah-Meir Caiserman, qui s'est distingué en explorant en langue yiddish les oeuvres poétiques associées à l'École littéraire de Montréal. Voulant mieux connaître les réalisations culturelles du Canada français, Caiserman a publié ses réflexions en 1921 dans une petite revue littéraire montréalaise intitulée Nyuansn. Ces contributions, peu étudiées jusqu'à maintenant, jettent un éclairage inédit sur le Québec d'avant la Révolution tranquille, et montrent tout l'intérêt qu'il y a à explorer les propos des auteurs issus des milieux allophones. 


\title{
H.-M. Caiserman et l'École littéraire de Montréal. Vers une exploration en yiddish du Canada français
}

\author{
Pierre ANCTIL \\ Département d'histoire \\ Université d'Ottawa
}

RÉSUMÉ - Au début du $X X^{e}$ siècle, un certain nombre d'immigrants européens ont jeté un regard sur la société québécoise et ont rédigé à ce sujet, à Montréal, des textes littéraires en langue non officielle. C'est entre autres le cas d'un activiste culturel et leader communautaire d'origine roumaine, Hananiah-Meir Caiserman, qui s'est distingué en explorant en langue yiddish les œuvres poétiques associées à l'École littéraire de Montréal.Voulant mieux connaître les réalisations culturelles du Canada français, Caiserman a publié ses réflexions en 1921 dans une petite revue littéraire montréalaise intitulée Nyuansn. Ces contributions, peu étudiées jusqu'à maintenant, jettent un éclairage inédit sur le Québec d'avant la Révolution tranquille, et montrent tout l'intérêt qu'il y a à explorer les propos des auteurs issus des milieux allophones.

ABSTRACT - At the beginning of the twentieth century, a number of European immigrants who had examined the specific features of Quebec society wrote, in Montréal, literary essays on the subject in non-official languages. Among them, a cultural activist and community leader of Romanian origins, Hananiah-Meir Caiserman, explored in Yiddish the poetry of the École littéraire de Montréal.To gain a better understanding of the cultural achievements of French Canada, Caiserman published his reflections in 1921 in a small Montréal literary journal, Nyuansn. His efforts, to which little attention has been paid to date, throw a new light on Quebec society before the Quiet Revolution and show the value of exploring the works of authors who belonged to linguistic minorities. 
$\mathrm{T}$

outes les sociétés reçoivent des immigrants venus de pays lointains, et le Québec moderne ne fait pas exception à cette règle. Parfois, lorsque les conditions s'y prêtent, les nouveaux citoyens s'intéressent de plus près à leur milieu d'accueil et consignent par écrit leurs observations au sujet des phénomènes qui les entourent. Dans certains cas, ces textes sont publiés outre-mer, où ils forment partie d'une littérature tournée vers l'exotisme et les voyages. Il arrive aussi que de telles réflexions paraissent en langue étrangère au Québec, c'est-à-dire dans des journaux ou des revues montréalaises destinées à des lecteurs immigrants encore peu à l'aise avec les langues officielles du Canada. Pour l'historien de l'immigration, ces témoignages et descriptions prennent un relief particulier, en ce sens qu'ils nous permettent de lire la réalité québécoise à travers un filtre culturel et politique entièrement différent, et où ne figurent pas les partis pris idéologiques qui ont marqué l'histoire canadienne depuis beaucoup plus longtemps. Le récit immigrant procède en effet le plus souvent de perceptions qui ne sont pas celles de la société dominante, et où le regard de l'écrivain appréhende d'une manière inédite le réel, comme s'il surgissait pour la première fois du néant. Le fait de cette rencontre soudaine et inattendue, imposée par le choc culturel issu de la migration, révèle des angles de lecture qui ont échappé à ceux qui se considèrent comme des natifs ou des résidants de longue date du pays. Fraîchement arrivés, les nouveaux venus se situent dans un espace de reconstruction et de recommencement propice à un positionnement créateur, ce qui leur permet de bénéficier d'un effet de distanciation difficilement accessible aux auteurs nés au pays.

Différent du récit du voyageur, qui ne fait par définition que passer brièvement en un lieu, le récit de l'immigrant s'arme d'une patience d'observation et d'une profondeur de sentiment peu communes. Cela tient à ce que s'y trouve inscrite la volonté de l'auteur d'appartenir et de participer à l'essor de la société d'accueil. À l'exception de textes rédigés en langue française ou en langue anglaise au $\mathrm{XX}^{\mathrm{e}}$ siècle, et qui émanaient pour la plupart de personnalités associées à des milieux cléricaux ou scientifiques, ce genre de littérature a été peu utilisé dans l’historiographie québécoise jusqu'à maintenant. Cela tient pour beaucoup à ce que les langues non officielles employées par les immigrants n’ont pas été suffisamment étudiées par les historiens du Québec, et que les œuvres littéraires rédigées dans ce contexte sont restées en grande partie inaccessibles aux chercheurs. On y découvre des réflexions utiles non seulement à l'histoire des communautés immigrantes elles-mêmes, mais aussi des propos significatifs touchant les 
relations que ces regroupements ont entretenues avec les francophones majoritaires, voire des observations sur l'ensemble de la société québécoise. Ces textes, rédigés du point de vue des nouveaux venus, révèlent aussi le degré d'ouverture et de tolérance à la diversité culturelle dont ont su faire preuve les différents milieux canadiens-français après le début de la grande migration européenne. De tels témoignages sont aussi représentatifs d'une volonté des immigrants de se porter au-devant du Montréal francophone, tel qu'ils le percevaient et le comprenaient, comme dans le cas qui nous intéresse ici.

Quelques années avant le début de la Première Guerre mondiale, un courant d'écriture en langue yiddish est apparu au Québec qui occupera pendant plusieurs décennies le devant de la scène au sein des différents milieux immigrants. Transposé depuis l'Empire russe et l'Europe de l'Est par une poignée de Juifs lettrés, cet élan littéraire comptait en son sein plusieurs individus aptes à rédiger au fil des ans le genre de témoignage et de descriptions que nous décrivions plus haut, et dont un en particulier retiendra notre attention: Hananiah-Meir Caiserman. Né en Roumanie, immigré à Montréal en 1910 à l'âge de 26 ans, Caiserman a été, en 1919, le premier secrétaire général du Congrès juif canadien. Quelques années plus tard, en 1934, il a repris le flambeau de l'organisme jusqu'à son décès en 1950. Caiserman avait aussi été un militant de la première heure à Montréal des syndicats de la confection et s'était illustré comme activiste des écoles de langue yiddish, de la Bibliothèque publique juive et des organisations sionistes de gauche. L'homme, qui avait un fort penchant littéraire, découvrit assez vite qu'il existait à Montréal d'autres traditions culturelles, elles aussi porteuses d'une subjectivité artistique digne d'intérêt pour un Juif récemment immigré au pays. Le sujet passionna tellement Caiserman qu'il se décida, quelques années après son arrivée au Canada, à parcourir attentivement les écrits des poètes de l'École littéraire de Montréal, reflet des aspirations nationales de sa société d'adoption. Caiserman apporta un grand soin à ce travail, au point où on peut $\mathrm{y}$ découvrir, près d'un siècle plus tard, une compréhension hautement originale du premier grand courant littéraire issu du Canada français.

Il ne faut pas se surprendre si la littérature yiddish montréalaise occupe une place importante dans les études sur l'immigration au Québec. Lieu de convergence culturelle très soutenu et espace de métissage exceptionnel, la littérature yiddish présente sur de longues périodes plusieurs interfaces avec des cultures et des langues non juives. Cette rencontre avec des parlers et des réalités appartenant à d'autres univers de signification 
était déjà, au moment de la grande migration vers le Canada, une tendance lourde du vécu culturel juif est-européen, à commencer par le contact avec les peuples de culture germanique, slave et balte ${ }^{1}$. Une des facettes les plus intéressantes de la recherche sur l'écriture yiddish montréalaise consiste d'ailleurs à mesurer jusqu'à quel point de telles tendances ont continué à avoir cours dans le milieu nord-américain, et par quelles voies elles se sont manifestées ${ }^{2}$. Dans le cas qui nous intéresse, il ne fait aucun doute que l'anglais a rapidement occupé une place de choix dans le quotidien des yiddishophones montréalais. Plusieurs témoignages éloquents situent dès le départ la langue de Shakespeare comme un passage obligé en vue d'une intégration réussie à la société canadienne, dont celui de Hershl Novak ${ }^{3}$ qui décrit dans ses Mémoires la situation faite aux jeunes immigrants yiddishophones arrivés à Montréal vers 1910. Comme l'explique Novak, les modalités de travail dans l'industrie de la confection et dans le commerce de détail, conjuguées aux pressions manifestées dans les institutions communautaires fondées par les Yahudim montréalais ${ }^{4}$, poussaient très rapidement les jeunes Juifs est-européens à se mettre d'abord à l'apprentissage de l'anglais.

Il reste que d'autres langues étaient aussi présentes dans l'environnement urbain qui s'étalait au pied du mont Royal, dont celle utilisée par la majorité démographique dans la ville: le français. C'était sans compter les parlers privilégiés par d'autres populations immigrantes moins importantes par le nombre, comme l'italien, le russe, l'ukrainien, le polonais et l'allemand, pour n'en nommer que quelques-uns. Plusieurs de ces langues étaient d'ailleurs connues des lettrés yiddishophones qui se sont dirigés vers le Canada, soit qu'ils les avaient étudiées dans leur ville d'origine, soit qu'ils les avaient côtoyées au sein de l'Empire russe ${ }^{5}$.

1. Jean Baumgarten, Le yiddish : histoire d'une langue errante (Paris, Albin Michel, collection Présence du judaïsme, no 26, 2002), $281 \mathrm{p}$.

2. Voir à ce sujet Chantal Ringuet, À la découverte du Montréal yiddish (Montréal, Fides, 2011), $300 \mathrm{p}$.

3. Voir à ce sujet Hershl Novak, La première école yiddish de Montréal, 1911-1914 (Sillery, Septentrion, 2009), 170. L'ouvrage est une traduction de Hershl Novak, Fun mayn yunge yorn [au sujet de mes années de jeunesse], (New York, 1957, Arbeter Ring bildungs-komitet), 159. Ces mémoires ont été traduites par l'auteur de cet article.

4. Ce terme hébraïque réfère aux premiers immigrants juifs montréalais d'origine britannique, et qui avaient l'anglais comme langue maternelle.

5. Voir à ce sujet les données compilées dans la traduction française du dictionnaire biographique de Haim-Leib Fuks, Cent ans de littérature yiddish et hébrä̈que au Canada (Sillery, Septentrion, 2005), p. 439. L'ouvrage en version originale yiddish, Haim-Leib Fuks, Hundert yor Yidishe un Hebreyshe literatur in Kanade (Montréal, publié à compte d'auteur, 1980), 326 p., a été traduit en français par l'auteur de cet article. 
Dans ce contexte de diglossie avancée, la question se pose d'emblée, à savoir quel avait été le degré de contact à Montréal, au début du $\mathrm{XX}^{\mathrm{e}}$ siècle, entre les élites culturelles de langue yiddish et celles réunies autour des institutions catholiques de langue française ${ }^{6}$ ? En Russie impériale, le français était considéré comme un des principaux véhicules de la culture savante et la langue était particulièrement prisée dans les cercles littéraires et scientifiques, une réalité à laquelle l'alliance politique entre la France de la Troisième République et l'Empire des Romanov donnait beaucoup de prestige. Qu'en était-il par contraste du contexte montréalais où le français possédait un niveau de visibilité que n'avaient pu manquer d'observer les immigrants juifs venus d'Europe de l'Est? Nous savons, grâce au dictionnaire biographique de Haim-Leib Fuks, que quelques auteurs yiddishophones montréalais pouvaient faire preuve d'une certaine aisance dans la langue de Molière, dont entre autres Reuben Brainin, BenjaminGutelius Sack ${ }^{7}$, Louis Benjamin et Joseph Kagedan (Kage), pour ne mentionner que les plus talentueux. Comme son compatriote d'origine rou-

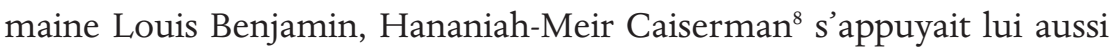
sur les origines latines de sa langue maternelle pour parcourir des textes en langue française.

Dans un milieu montréalais fortement compartimenté sur le plan linguistique et religieux, qu'en était-il de ces quelques passerelles qui pouvaient laisser croire à une rencontre possible entre lettrés yiddishophones et canadiens-français ? Certes, à Montréal, francophones et parlants yiddish se côtoyaient sans cesse dans les usines de confection et au sein des commerces de proximité du boulevard Saint-Laurent, mais ces rapports touchaient surtout les classes laborieuses et n'eurent pas de retombées notables sur le plan littéraire. Y avait-il au XX $\mathrm{XX}^{\mathrm{e}}$ siècle une ouverture suffi-

6. Voir à ce sujet la discussion menée par l'auteur de cet article dans Pierre Anctil, «Les lettres yiddish au Canada; des immigrants prennent la plume», Trajectoires juives au Québec (Québec, Les Presses de l’Université Laval, 2010), 146.

7. Sack s'intéressa d'un point de vue historique à la période de la Nouvelle-France. Il publia un certain nombre de textes à ce sujet en 1926 dans l'ouvrage dirigé par Arthur Daniel Hart: The Jew in Canada, $a$ Complete Record of Canadian Jewry from the Days of the French Régime to the Present Time (Toronto et Montréal, Jewish Publications, 1926), 575 p. La plupart de ces études étaient basées sur des documents d'époque rédigés en français. Sa contribution principale a paru en 1948: Benjamin-Gutelius Sack, Geshikhte fun Yidn in Kanade, fun di friste onhayb biz der letster tsayt [1'histoire des Juifs au Canada depuis les premiers temps jusqu’à la période contemporaine] (Montréal, publié à compte d’auteur, 1948), 358 p. On en trouve une version anglaise sous le titre History of the Jews in Canada (Montréal, Harvest House, 1964), 299 p.

8. Voir à ce sujet Pierre Anctil, «H. M. Caiserman ou la passion du yiddish», Tur Malka, flâneries sur les cimes de l'histoire juive montréalaise (Sillery, Septentrion, 1997), 75-107. Voir aussi Pierre Anctil, Sherry Simon et Norman Ravvin, dir., New Readings of Yiddish Montreal/Traduire le Montréal yiddish (Ottawa, Presses de l'Université d'Ottawa, collection «Études canadiennes », 2007), 125 p. 
sante, au sein des milieux lettrés francophones et yiddishophones, pour qu'un processus d'échange, même timide, s'établisse entre ces deux grandes traditions culturelles?

Longtemps les chercheurs ont cru que les rapports entre intellectuels canadiens-français et Juifs est-européens ressemblaient aux vastes étendues enneigées qu'une brise glaciale forme en ce pays chaque hiver. Ultramontaine, traditionaliste et d'inspiration française classique, l'élite cléricale du Québec avait été éduquée dans un esprit de méfiance face au judaïsme, et considérait souvent les relations avec les Juifs montréalais comme problématiques. Nouvellement arrivés, de culture cosmopolite et jugés plus susceptibles d'embrasser la langue anglaise, les immigrants est-européens donnaient l'impression de former une sorte d'interstice à la frontière entre les deux grands ensembles linguistiques présents dans la région montréalaise. Éduqués dans le giron de l’Église, plusieurs écrivains, artistes et penseurs francophones avaient fini par développer face aux Juifs une attitude de mépris lointain qui ressemblait le plus souvent à de l'indifférence, et parfois prenait les couleurs d'un antisémitisme doctrinal inspiré de Rome. Les historiens ont jugé que peu d'empathie mutuelle était susceptible de se développer sous un tel climat, d'autant plus que nul ne savait distinguer très bien chez les francophones entre les littératures hébraïque et yiddish modernes, entre l'orthodoxie judaïque et les courants progressistes laïques ou entre le sionisme et le nationalisme diasporique.

En somme, peut-être faute d'avoir vraiment fouillé la question, les spécialistes ont conclu qu'il fallait attendre la fin des années 1930 pour voir poindre au Canada une première lueur de dialogue entre Juifs et francophones, le plus souvent sous la menace d'événements extérieurs, comme la venue au pouvoir d'Hitler en Allemagne et la crainte d'une nouvelle guerre mondiale, sans oublier en corollaire la montée de l'antisémitisme au pays. En somme, au moins trente ans s'étaient écoulés entre le début de la grande migration est-européenne et l'établissement d'une première écoute entre les deux groupes, où la langue et la culture yiddish allaient jouer un rôle négligeable.

Ce point de départ je l'avais en tête lorsque j'ai entrepris une recherche Killam sur la carrière du poète yiddish montréalais Jacob-Isaac Segal (18961954). Mon objectif était de rédiger une biographie complète de l'écrivain, qui couvrirait les premières années de sa vie en Ukraine, son immigration au Canada en 1910, son séjour à New York au cours des années 1920 et la 
parution de ses derniers recueils rédigés à Montréal après la guerre ${ }^{9}$. Pour y parvenir, j’ai pris la décision de consulter en détail les archives de la Bibliothèque publique juive, où les manuscrits et la correspondance du poète avaient été déposés, ainsi que celles du Congrès juif canadien ${ }^{10}$, qui contenaient les documents personnels de Hananiah-Meir Caiserman. Le parcours allait s'avérer long et ardu, mais il réunissait la plupart des textes et témoignages susceptibles d'éclairer la carrière de Jacob-Isaac Segal. Je savais que Segal et Caiserman étaient liés par une amitié très significative sur le plan historique, et que ce dernier avait recueilli à de nombreuses reprises au cours de sa carrière les confidences intimes du poète. J'ai ainsi eu l'occasion de constater que les deux hommes s'étaient connus dans un contexte très particulier, alors que Segal publiait ses premiers poèmes vers 1915-1917 dans le Keneder Odler [l'Aigle canadien], le quotidien yiddish de Montréal, et qu'il émergeait à peine sur la scène littéraire yiddish de Montréal. Les poèmes de Segal, qui était alors un jeune homme au début de la vingtaine, avaient été remarqués par un cercle de lecteurs enthousiastes animé par Moshe Shmuelsohn et gravitant autour de la Montreal Shrayber Farayn [l'association des écrivains yiddish de Montréal], dont Caiserman lui-même faisait partie.

Sauf que nul ne savait au juste, à l'époque, qui était ce Jacob-Isaac Segal. Il venait timidement déposer ses textes à l'Odler puis disparaissait sans laisser de traces. Même Hershl Hirsch, le directeur du journal, ignorait quelle allure le poète pouvait avoir. Jusqu'à ce que l'on découvre que le prodige littéraire en question était un jeune ouvrier qui appartenait au syndicat des tailleurs... dont Caiserman était président depuis plusieurs années. Voyant cela, les membres du Montreal Shrayber Farayn réussirent à convaincre Segal de participer à une soirée littéraire qui dut avoir lieu en 1917. Pour la première fois, ils purent entendre le poète faire lecture de son œuvre et révéler l'étendue de son talent. L’année suivante, en 1918, sans doute grâce au soutien moral et financier des membres du Farayn, Segal réussit à publier un recueil de poèmes intitulé Fun mayn velt [de mon univers], le premier à paraître dans cette langue à Montréal.

Ces événements montrent bien le rôle de premier plan que Caiserman joua lors de la naissance de la littérature yiddish canadienne, à tel point que le poète lui en resta reconnaissant pour le reste de ses jours. Au cours

9. Cette biographie a paru sous le titre: Pierre Anctil, Jacob-Isaac Segal (1896-1954), un poète yiddish de Montréal et son milieu (Québec, Les Presses de l’Université Laval, 2012), 433 p.

10. Ces archives portent aujourd'hui officiellement le nom d'Archives nationales du Congrès juif canadien, (ANCJC), Comité des charités. 
des années suivantes, Caiserman développa un tel intérêt pour l'écriture yiddish, qu'il devint dans les faits le premier critique littéraire à apparaitre dans cette langue au Canada. Tout au long des années 1920 et 1930, il suivit de très près les progrès de l'écriture yiddish au pays et demeura en contact constant avec la plupart de ses artisans, dont Jacob-Isaac Segal. De fait, Caiserman fit tant et si bien qu'en 1934 il publiait à Montréal la première anthologie de la poésie juive canadienne, intitulée Yidishe dikhter in Kanade [les poètes juifs au Canada], laquelle contenait les textes de près de quarante écrivains, autant en langue yiddish qu'en langue anglaise. Nous savons aussi, par les archives du Congrès juif canadien, que Caiserman caressait l'idée au cours des années 1940 d'écrire en yiddish une biographie détaillée de son ami Segal, projet qui s'échoua probablement sur les écueils d'une vie communautaire et politique trop remplie.

C’est à la suite de ces découvertes que j'eus le désir de parcourir de fond en comble les documents littéraires déposés par Caiserman aux archives du Congrès juif canadien. Manifestement, Caiserman avait non seulement été un témoin oculaire de l'efflorescence de la littérature yiddish canadienne, mais il avait en plus été le premier à réfléchir au sens qu'il convenait de donner à ce phénomène, autant sur le plan culturel que politique. En réalité, Caiserman avait fait beaucoup plus que soutenir par tous les moyens possibles son ami Jacob-Isaac Segal et quelques autres auteurs apparus dans son sillage. Dès le début des années 1920, il avait pris la plume pour hâter au pays l'apparition d'une littérature yiddish d'envergure et pour paver la voie à son émergence sur la scène internationale.

Cet engagement de tous les instants est palpable dans un essai précoce que Caiserman fit paraître au début de l'année 1921 dans la revue montréalaise Nyuansn [nuances] $]^{11}$, intitulé «Kanade [Canada $]^{12}$ », et qui portait sur les lettres yiddish canadiennes. Dans ce texte, on trouve plusieurs éléments en vue d'un positionnement de l'écriture en langue yiddish au pays, dont au premier chef l'importance pour les poètes immigrants de s'attacher à décrire et à chanter leur nouvelle patrie. Le paysage canadien entre autres, si majestueux et si imposant, qui s'étendait inexploré sur d'immenses territoires, méritait dans l'esprit de Caiserman toute l'attention des auteurs d'origine est-européenne. Comment la littérature yiddish

11. La revue Nyuansn ne parut que trois fois à Montréal au cours de l'année 1921. Animée par JacobIsaac Segal et de haute volée littéraire, elle publia surtout de la poésie, des essais culturels et quelques courts récits.

12. Hananiah-Meir Caiserman, «Kanade», Nyuansn, 1, (janvier 1921): 15-17 et «Kanade», Nyuansn (février 1921): 36-38. 
pourrait-elle prétendre au titre de canadienne, si elle détournait le regard de ces réalités et refusait de prendre en compte la vastitude du continent, la rigueur de son climat et le mode d'adaptation de ses premiers habitants? Et que dire de l'histoire du pays, de ses commencements sous le Régime français, puis plus tard de son passage au statut de dominion britannique. N’y avait-il pas là une invitation adressée aux écrivains et artistes yiddishophones, qui étaient maintenant conviés à se joindre à leur société d'accueil, à en reconnaître les mérites et à contribuer par leurs talents à son avancement? Immigré au Canada depuis à peine dix ans, Caiserman propose dans «Kanade» un constat très positif, qui est aussi une exhortation faite aux écrivains yiddish à prendre part intensément, dans leur langue, à la vie culturelle de leur nouveau pays :

Kanada, di yunge Kanada, mit ir merkvirdiger industryel-ekonomisher tetigkayt, mit ir sotsyal-politisher unruhigkayt, mit ir fantastish-poetisher geografisher lage un nit-bagrenitstkayt fun natur-vunder un klimatn, dos kosmopolitishe land mit sharfe natsyonale problemen, dos brayt, vild-mild Kanada, vos iz azoy mesugl tsu der bashafung fun a raykher literatur ${ }^{13}$.

Or pour parvenir à se situer dans ce pays éminemment prometteur, mais si inhabituel par sa taille géographique et l'ampleur de ses richesses naturelles, les écrivains yiddish se devaient aussi de parcourir les accomplissements littéraires de leurs devanciers canadiens-français et canadiensanglais. Comment en effet les poètes d'origine est-européenne pourraientils atteindre au sommet des lettres canadiennes, s'ils ne connaissaient rien des thèmes que les auteurs nés sur cette terre avaient déjà développés, ni des émotions dont ils avaient été saisis à la vue d'une nature sauvage inaltérée par la présence humaine? Bien avant eux, des communautés s'étaient établies le long des fleuves et des rivières que compte le Canada. Dans ces campagnes et bourgades, des hommes et des femmes avaient entrepris de découvrir à travers l'écriture l'ampleur du pays, la beauté de ses paysages et le mystère de ses saisons. Ils avaient aussi goûté au dur labeur des champs, engrangé des récoltes abondantes et exploré de vastes régions. Les Canadiens français en particulier, devenus minoritaires après la Conquête anglaise, s'étaient affirmés grâce aux belles-lettres et avaient choisi de préserver leur langue en produisant des œuvres poétiques mar-

13. «Le Canada, le jeune Canada, avec sa remarquable activité industrielle et économique, avec son agitation sociale et politique, avec sa géographie fabuleusement poétique, avec ses innombrables merveilles naturelles et son climat si varié ; pays cosmopolite avec ses intenses problèmes nationaux, ce vaste, ce sauvage et doux Canada, si propice à la création d'une riche littérature », H.-M. Caiserman, «Kanada », Nyuansn, janvier 1921, loc. cit., 15. 
quées par la canadianité. Précurseurs en ce pays, ils deviennent pour Caiserman des modèles à suivre dans l'affirmation d'une identité juive distincte, sans cesse renouvelée par l'invention d'une écriture originale et porteuse d'une émotion artistique de haut calibre. Traversant ce paysage imaginaire de la littérature canadienne-française, Caiserman a en effet découvert:

A gantse galerye Keneder frantsoyzisher dikhter, fun vemen dikhtung es otemt aroys di intim-vild-farshayte Keneder atmosfere, di perzenlikhe aspiratsyes fun Keneder tip mentsh un di nebelhafte troymerayen fun dem natsyonal-religyez-mistishen klal ${ }^{14}$.

L'apparition d'une tradition littéraire au sein du Canada français amène de plus Caiserman à constater que les francophones, plus que les anglophones, projetaient leurs aspirations nationales dans un ordre d'idée supérieur, soit dans la sphère littéraire, artistique et spirituelle. En agissant ainsi, ils assuraient la pérennité de leur culture dans le contexte nordaméricain et tissaient les liens qui uniraient, en situation de crise politique, le peuple à ses élites politiques et religieuses. Comment pouvait-il en être autrement, se demandait Caiserman: «Di Keneder frantsoyzen, di aygentimlikhe bal'abatim fun dem rizigen Kanade, hoben do far yor-hunderte gekemft un geliten, un aroyfgelegt dem shtempel fun zayer etnisher individualitet oyf'n gantsn Keneder leben ${ }^{15}$.» Ces pages remarquables par leur précocité historique et par la connaissance de la littérature canadiennefrançaise qu'elles supposaient, situent d'emblée Caiserman comme le plus grand critique immigrant de sa génération.

L'article paru en 1921 dans Nyuansn révèle le parcours étourdissant de Caiserman à travers les lettres francophones du Canada. Il dénote de plus pour la première fois un approfondissement inespéré de ces thèmes au sein de la communauté yiddishophone montréalaise. Manifestement, Caiserman maittrise le sujet et ne ressent aucune hésitation à discourir sur lui, entre autres sur le plan de la périodisation, des thèmes traités et de l'originalité de la littérature canadienne-française par rapport à son équivalent européen français. L'activiste connaît aussi, pour les avoir de toute évidence parcourus, des auteurs assez peu diffusés en dehors des milieux

14. "Tout un florilège d'écrivains canadiens-français, qui laissaient transparaître dans leur poésie l'atmosphère intime, sauvage et imprévisible du Canada ainsi que les aspirations personnelles du type d'homme canadien. Dans ces œuvres on pouvait percevoir d'une manière encore idéaliste et imprécise l'élan national, religieux et mystique du peuple», ibid., 16.

15. «Les Canadiens français, les véritables occupants de ce vaste Canada, ont lutté et combattu pendant des siècles sur son territoire. Ils ont laissé l'empreinte de leur ethnicité sur l'ensemble de la vie canadienne", id. 
francophones, ou dont les publications se limitaient à quelques opuscules édités à un moment où les belles-lettres étaient encore naissantes au Canada français:

Dos erklert take farvos di Keneder-frantsoyzen kenen onvayzen oyf aza groyse tsol poeten fun farshidenartigste rikhtungen velkhe bazingen oyf aza oder anderen oyfn di shtrebungen fun zayer folk: Azoy tsum bayshpil hoben ze shoyn in der tsayt fun 1860-1879 gehat 3 vikhtige dikhter velkhe zingen hoyptzakhlikh vegen di natsyonale shtrebungen fun folk, nemlikh, F. Garneau, Joseph Lenoir un der folkstimlikhter fun ze, Octave Crémazie. Abisel shpeter azelkhe liriker vi Lemay un Adolphe Poisson un romantishe mistiker, vi: Gingras un Émile Nelligan ${ }^{16}$.

Confronté à l'apparition au Canada, au milieu du XIX ${ }^{e}$ siècle, d'une littérature francophone d'inspiration nationale, chantant les beautés naturelles du pays et faisant la promotion d'une culture minoritaire, Caiserman n'hésite pas un seul instant à voir dans ce mouvement un exemple à suivre pour les lettres yiddish canadiennes. De fait, Caiserman avait côtoyé et parcouru la production littéraire de langue française, justement parce qu'il était devenu convaincu que les poètes canadiens-français montraient la voie à leur vis-à-vis de langue yiddish. En résistant à la conquête anglaise, en bravant l'hégémonie culturelle britannique que Londres avait tenté d'établir dans la colonie et en restant à l'écoute des classes laborieuses, ces écrivains étaient devenus les symboles de leur nation.

Certes la situation du Canada français au XIX ${ }^{e}$ siècle différait sous plus d'un rapport de celle des yiddishophones canadiens - notamment par le fait que les francophones référaient le plus souvent à un univers de réalités rurales, tandis que les Juifs étaient au contraire des urbains et souvent des travailleurs industriels - mais dans l'esprit de Caiserman, les deux peuples étaient animés d'un commun désir de libération et ils cherchaient à mettre de l'avant une langue menacée de toutes parts. Et quelle meilleure manière de soutenir le français et le yiddish, sinon en créant de toutes pièces une littérature qui deviendrait le véhicule privilégié du devenir national. Moins influents dans la sphère économique et politique que les anglophones, les Canadien français n'en avaient pas moins suscité l'apparition de nombreux mouvements littéraires au pays, et surtout ils s'étaient aventurés hors des

16. "Cela explique sans doute pourquoi les Canadiens français peuvent d'enorgueillir d'un aussi grand nombre de poètes si différents les uns des autres, qui chantent d'une manière ou d'une autre les aspirations de leur peuple: par exemple, ils avaient déjà au cours de la période 1860-1879 trois poètes importants qui reflétaient principalement les ambitions nationales du peuple, c'est-à-dire [François-Xavier] Garneau, Joseph Lenoir et le plus sensible aux désirs du peuple, Octave Crémazie. Un peu plus tard se manifestèrent des poètes lyriques comme [Pamphile] Lemay et Adolphe Poisson, ainsi que des romantiques à saveur mystique, tel [Apollinaire] Gingras et Émile Nelligan», ibid., 17. 
sentiers battus en valorisant leur identité culturelle: "Genug dem fakt tsu konstatiren az oykh do in Kanada iz bashtetigt gevoren der ongenumener klal az dort vi a minoritet firt on an aktiven kamf far zelbst-derhaltung, muz zi bashafen naye formen fun literatur ${ }^{17}$.»

En somme, le Canada français montrait la voie aux lettrés yiddish qui venaient à peine de faire leur entrée au Canada et qui, de l'avis de Caiserman, insistaient trop souvent pour reprendre dans leurs œuvres poétiques des thèmes qui relevaient des expériences vécues dans l'Ancien Monde.

Des recherches plus approfondies, menées à la fin de 2008 dans les archives du Congrès juif canadien, montrent que l'article de 1921, publié dans Nyuansn, et l'ouvrage de 1934 paru sous le titre de Yidishe dikhter in Kanade, ont d'abord existé sous la forme d'un manuscrit de 140 pages en yiddish dont la première ligne est comme suit: «O, Kanada! O, Kanada!... mit di bavunderung-verter hoybt zikh on der natsyonaler himn ${ }^{18} \ldots$. Ce document, non daté, non titré et écrit à la main par Caiserman sur une longue période ${ }^{19}$, contient sous une forme embryonnaire l'essentiel de la pensée du critique littéraire au sujet des belles-lettres yiddish canadiennes, et dont seule une partie a été publiée subséquemment. Le projet à l'origine se voulait une analyse de la poésie canadienne de langue anglaise (p. 7-18), de langue française (p. 18-30) et de langue yiddish (p. 30-140), et s'est probablement poursuivi de 1919 jusqu'au début des années $1930^{20}$. Une partie importante des douze premières pages du manuscrit a paru en 1921 dans Nyuansn, puis les cent dix dernières pages ont formé en 1934 l'essentiel du texte de Yidishe dikhter in Kanade, augmenté d'une préface de JacobIsaac Segal, d'une introduction de l'auteur et d'un chapitre sur la poésie juive canadienne de langue anglaise.

Par contre, dans ce manuscrit, l'argumentaire principal de Caiserman au sujet de la poésie canadienne-anglaise et canadienne-française était resté à ce jour inédit, sans doute parce que l'auteur jugeait qu'il ne serait pas d'un intérêt capital pour son lectorat de langue yiddish. Dans ce seg-

17. «Il suffit de constater que la règle générale se confirme ici au Canada, à savoir que là où une minorité mène une lutte intense pour son affirmation (nationale), elle se doit aussi de créer de nouvelles formes littéraires", id.

18. "O Canada!, O Canada!, c'est avec ces mots merveilleux que s'ouvre l’hymne national...». "O Kanada! O Kanada!...», ANCJC, Comité des charités, DA 1/ 6/2, 21.

19. Le manuscrit est couché sur des feuilles de papier d'une texture et d'un format très différents, ce qui donne à penser que l'écriture de Caiserman était entrecoupée de pauses assez longues, notamment lorsque l'auteur a fait un séjour en Palestine de juillet 1921 à novembre 1923.

20. En page 82, le manuscrit contient notamment une mention de l'œuvre de Jacob-Isaac Segal intitulée Lirik [lyrique], publiée à Montréal en 1930. 
ment de texte, le critique littéraire développe sa compréhension des lettres canadiennes-françaises et canadiennes-anglaises et aborde les œuvres de plusieurs écrivains anglo-britanniques connus à l'époque, comme E. Pauline Johnson, Archibald Lampman, Bliss Carman, Duncan Campbell Scott et Marjorie Pickthall. Il jette ensuite un regard sur leurs équivalents francophones, entre autres Pamphile Lemay, Louis Fréchette, Alphonse Beauregard et Paul Morin, avec une mention explicite de l'École littéraire de Montréal ${ }^{21}$. Dans plusieurs cas, Caiserman se permet de longues citations en anglais ou en français, notamment des poèmes rédigés par Pamphile Lemay, François-Xavier Garneau, Alphonse Beauregard et Paul Morin, tous transcrits dans une calligraphie impeccable et respectant dans ses moindres détails l'orthographe correcte du français, incluant les accents, les cédilles, les points-virgules et les majuscules. Manifestement, Caiserman avait lu et relu les poètes canadiens-français dans le texte et s'était procuré les rares études sur le sujet qui avaient été publiées à cette époque, dont sans doute les ouvrages de l'abbé Camille Roy parus quelques années auparavant ${ }^{22}$.

Cette exploration étonnante d'une littérature, dont Caiserman ignorait sans doute l'existence avant son arrivée au Canada, surprend d'autant plus qu'elle exigeait une fine connaissance linguistique du français et de l'anglais, dont peu d'immigrants yiddishophones étaient capables au début $\mathrm{du} \mathrm{XX}^{\mathrm{e}}$ siècle. L'empressement de Caiserman à surmonter des barrières culturelles, toujours imposantes à l'époque, exprimait aussi chez lui un désir de canadianisation et de pleine participation à la vie littéraire du pays qui ne s'était guère manifesté dans les rangs des écrivains yiddish montréalais jusque-là.

Emporté dans sa quête d'une littérature nationale canadienne-française et pressé de suivre les traces de ses principaux chantres, Caiserman s'aventura même à franchir une frontière restée jusque-là impénétrable, c'est-

21. En réalité, Caiserman nomme une vingtaine d'auteurs canadiens-français de plus dans son manuscrit, divisé en quatre segments historiques, mais sans s'arrêter à leur œuvre spécifiquement. Il s'agit dans l'ordre de: Jacques Viger, Michel Bibaud, Joseph-Édouard Turcotte; Pierre-Joseph-Olivier Chauveau, Octave Crémazie, Gonzalve Desaulniers, Adolphe Poisson, Eudore Évanturel, Nérée Beauchemin; Émile Nelligan, Albert Lozeau, Charles Gill, Engelbert Gallèze, Jean Charbonneau, Alonzo Cinq-Mars, Émile Vézina, Albert Ferland, Charles Daoust, Germain Beaulieu; René Chopin, Benjamin Michaud, Édouard Chauvin, Jean Nolin, Roger Maillet, Émile Venne, Blanche Lamontagne-Beauregard et Alfred Garneau. Caiserman mentionne aussi Madeleine de Verchères, Dollard des Ormeaux et LouisJoseph Papineau comme des «héros nationaux» du Canada français.

22. On pense ici à deux études publiées à Québec en 1907, Tableau de l'histoire de la littérature canadienne-française (Québec, Imprimerie de l'Action sociale, 1907) et Essais sur la littérature canadienne-française (Québec, Garneau, 1907) ainsi qu’à Nos origines littéraires (Québec, Imprimerie de l’Action sociale, 1909). 
à-dire qu'il traduisit quelques extraits poétiques du français vers le yiddish. Sans doute le premier yiddishophone à tenter ce passage, Caiserman jeta son dévolu sur Pamphile Lemay, considéré au début du XX $\mathrm{X}^{\mathrm{e}}$ siècle comme un des plus grands poètes canadiens-français et décrit par le critique littéraire lui-même comme: «a lirisher folks-dikhter durkh un durkh, farlibt in der kanader natur un folk un in zayne varemste natsyonale un religyeze shtrebungen ${ }^{23}$ ». Lemay incarnait probablement dans l'esprit de Caiserman les principales qualités que les poètes yiddish se devaient d'adopter dans leur pays d'adoption, soit une admiration sans bornes pour le paysage canadien, un amour intense de la patrie et une sensibilité aux accents particuliers de la langue populaire. Ce parti pris se reflète d'ailleurs dans les extraits que Caiserman choisit de traduire, qui portent sur la récolte aux champs, sur le caractère majestueux du fleuve Saint-Laurent et sur l'émigration des Canadiens français vers les États-Unis, tous tirés du recueil de Lemay paru en 1914 et intitulé Les épis. Deux exemples suffiront à convaincre le lecteur contemporain de l'audace de Caiserman:

$O$, di shtarke lider vos hoybn zikh fun di prairies

Di vunderlikhe reykhes fun tsaytign hay

$O$, di tsvaygn mit blonden, di grine draperyen

Vos vign zikh un shvebn in bloyen azur ${ }^{24}$.

$[\ldots]$

Oyb du volst gekent in dayn kishef vander

$O$, vunderlikher taykh, $O$ liber vagabond

Volstu dertsaylt undz, far vos azoy noent fun dayn shtam farlirstu zikh in tifn yam ${ }^{25} \ldots$

Les traductions de Lemay ont dû être faites par Caiserman à la fin des années 1910, à une époque où très peu de lecteurs yiddishophones s'étaient encore intéressés d'aussi près à la vie littéraire canadienne-française. Elles constituent, de ce fait, une étape décisive dans l'évolution de la littérature canadienne d'expression yiddish. En lisant avec autant d'in-

23. "Un poète lyrique enraciné dans l'élan populaire, épris de la nature canadienne et attentif aux aspirations nationales et religieuses profondes du peuple canadien», "O, Kanada ! O, Kanada !...», ANCJC, Comité des charités, fonds Caiserman, DA 1/6/2, 21.

24. «O les vives chansons qui montent des prairies!/ L'exquise senteur du foin mûr!/Ô les rameaux en fleurs, les vertes draperies/Qui flottent sous un ciel d'azur!», Pamphile Lemay, «La fenaison», Les épis (Montréal, J.-Alfred Guay, 1914), 33.

25. «Si tu pouvais parler dans tes vaillantes courses, /Ô fleuve merveilleux!Ô fleuve vagabond!/Tu nous dirais pourquoi loin, bien loin de tes sources, / Tu vas enfin te perdre à l'océan profond ", Pamphile Lemay, "Si tu pouvais parler», Les épis (Montréal, J.-Alfred Guay, 1914), 68. Le troisième extrait traduit par Caiserman est tiré de Pamphile Lemay, «Dulcia linquimus arva», Les épis (Montréal, J.-Alfred Guay, 1914), 45. 
tensité un autre corpus littéraire canadien, en l'occurrence celui de langue française, Caiserman ouvrait la voie pour la génération de la grande migration à un enracinement au pays. Par ses efforts, Caiserman faisait la preuve qu'il était possible et souhaitable, même pour les Juifs est-européens, de se pénétrer des thèmes qui avaient retenu l'attention des poètes de langue officielle. Il y a fort à parier toutefois que Caiserman entreprit seul cette tâche. Tel un précurseur qui emprunte un chemin pour la première fois, le critique littéraire faisait découvrir à son modeste auditoire de 1921 un univers culturel aux multiples ramifications historiques et politiques. Il est fort probable aussi que Caiserman compléta ce périple littéraire sans rencontrer les principaux représentants des lettres canadiennes-françaises et sans pénétrer vraiment dans les cercles culturels francophones.

Rien dans son manuscrit ne laisse croire qu'il se serait porté au-devant de ces milieux et il n'existe aucune correspondance qui attesterait d'un échange épistolaire avec au moins un représentant de l'élite culturelle de langue française. En cette période du début du siècle, la présence juive est-européenne était encore trop récente à Montréal pour permettre de tels contacts, et le cloisonnement ethno-religieux encore trop étanche dans la ville, même si déjà à cette époque l'on jouait du théâtre yiddish au Monument national, boulevard Saint-Laurent. Pour l'essentiel, l'enthousiasme de Caiserman pour la poésie canadienne-française demeura un fait d'armes personnel, et ses connaissances en la matière purement livresques.

Les écrivains yiddish de Montréal mirent beaucoup de temps à franchir la distance culturelle que Caiserman avait balisée dans son article de 1921. Jeunes et inexpérimentés, les quelques poètes yiddishophones que comptait la ville au cours des années 1920 et 1930 luttaient déjà de toute leur énergie pour se tailler une place dans les cercles littéraires juifs locaux, et souvent leurs efforts étaient tournés vers les milieux new-yorkais ou, plus loin encore, vers leur continent d'origine. Simples ouvriers de la confection ou enseignants à temps partiel dans des écoles de langue yiddish, ils avaient aussi fort à faire pour gagner leur vie et nourrir leur famille. Plusieurs de ces auteurs écrivaient à l'occasion pour le Keneder Odler, ou pour des périodiques yiddish américains et, à part Jacob-Isaac Segal qui avait déjà publié cinq recueils de poésie en 1930, ils ne connurent pas toujours le succès dans l'entre-deux-guerres.

Surtout, les auteurs yiddish de Montréal peinaient pour la plupart à maîtriser les langues officielles canadiennes, en particulier le français, et 
ils auraient été pour la plupart incapables à cette époque d'accompagner Caiserman dans son exploration de l'École littéraire de Montréal. L’homme, il faut l'admettre, possédait une rare faculté d'ubiquité linguistique. De langue maternelle yiddish et éduqué en roumain à l'Académie commerciale de Bucarest, Caiserman avait appris l'allemand en pratiquant son métier de comptable en Europe de l'Est. Ce n'est que beaucoup plus tard, lors de ses nombreuses pérégrinations, qu'il s'était frotté au français, à l'anglais et à l'hébreu moderne. On peut d'ailleurs faire l'hypothèse qu'une fois installé à Montréal, Caiserman avait beaucoup mieux maîtrisé l'anglais que le français ${ }^{26}$. Contrairement à lui, la majorité des écrivains yiddish de Montréal étaient si attachés à leur société d'origine qu'ils mirent au moins vingt ans de plus avant de se convaincre de vraiment tourner le regard vers la nature ou la vie canadienne. Ce virage se produisit en fait au lendemain de l'Holocauste, quand il devint évident aux yeux des lettrés yiddishophones que l'univers dont ils étaient issus avait disparu pour toujours, comme le montre ce témoignage éloquent du poète montréalais Sholem Shtern ${ }^{27}$ :

En 1949, après ma première visite dans la Pologne d'après-guerre, une transformation s'est opérée en moi. J'eus alors l'occasion de voir de près les conséquences de l'Holocauste, et de constater comment étaient disparus pour toujours les petites localités et certains quartiers urbains qui débordaient de vie juive. Les nazis avaient détruit la culture et la créativité juive en Pologne, autant pour ce qui était de ses aspects traditionnels que de ses composantes plus modernes. Tout un mode juif se trouvait réduit en cendres. Les grands centres juifs de Pologne, comme Lublin, Lemberg (Lvov), Cracovie, Zamosc, Vilnius et Varsovie, où brillait la lumière de la Tora et d'où avaient rayonné autant les œuvres immémoriales que celles plus récentes du judaïsme, plus jamais ne seraient une source d'inspiration pour nous $[\ldots]$.

Il s'agit là de la cause principale qui m’a poussé à quitter les chemins depuis longtemps parcourus. Voilà comment $j$ 'ai décidé de passer la plus grande partie de ma vie dans un pays nouveau, même si je ressentais encore sous une forme nostalgique l'attraction de ma ville natale, pour résolument m'attacher à décrire l'existence telle qu'elle se déroulait en Amérique ${ }^{28}$.

26. Dans une lettre à J.-A. Foucher, de Shawinigan Falls, Caiserman avouait le 4 mai 1942: «I am in receipt of your interesting letter of May $1^{\text {st }}$, and I am answering it in English for I can express myself much better». ANCJC, Comité des charités, fonds Caiserman.

27. Originaire de Pologne, Sholem Shtern était arrivé à Montréal en 1927. Son premier recueil de poésie canadien, intitulé Noentkayt [proximité], avait été publié à Toronto en 1929.

28. Sholem Shtern, Nostalgie et tristesse. Mémoires littéraires du Montréal yiddish (Montréal, les Éditions du Noroît, 2006), 260. Il s'agit d'une traduction partielle des Mémoires de l'auteur parus à Montréal en 
L'intuition novatrice de Caiserman face à la littérature canadienne d'expression française et ses premières traductions du français vers le yiddish nous obligent à revoir une partie de la chronologie qui avait cours jusqu'à maintenant au sein des études juives canadiennes. De fait, à la lumière de ces éléments restés jusqu'ici inconnus, il nous faut avancer d'au moins dix ans les premiers efforts de Caiserman pour se porter à la rencontre du Canada français. Longtemps, j’ai cru que le critique littéraire s'était mobilisé en vue d'un dialogue avec ses compatriotes francophones, surtout parce qu'il craignait l'influence sur eux de l'antisémitisme doctrinaire de l'Église catholique et de l'idéologie nazie. Une fois installé en 1934 aux commandes du Congrès juif canadien, soit au lendemain de la prise de pouvoir d'Hitler en Allemagne, Caiserman avait multiplié les tentatives d'ouverture vers les milieux canadiens-français de tendance plus libérale, notamment certaines maisons d'enseignement jésuites à Montréal ${ }^{29}$. Or sa volonté de traverser la barrière linguistique et religieuse qui séparait les yiddishophones et les Canadiens français daterait plutôt de la fin des années 1910, comme le montre de manière éloquente le manuscrit décrit plus haut.

Cette avancée de la recherche éclaire aussi une période ultérieure et explique en grande partie pourquoi Caiserman a été si sensible aux aspirations politiques des francophones ${ }^{30}$, notamment quand s'enclenche au début des années 1930 une première tentative de dialogue interreligieux avec le père jésuite Joseph Paré. Pour avoir remis en perspective, dès les années 1910, des écrivains comme Louis Fréchette, Émile Nelligan et Pamphile Lemay, Caiserman comprenait mieux vingt ans plus tard les racines historiques et émotionnelles du nationalisme canadien-français. À défaut de saisir en détail les rouages internes de l'Église catholique et de la vie politique montréalaise, il avait senti que ce courant d'idée s'enracinait dans un terroir vieux de près d'un siècle. Sans ces premières explorations révélatrices de l’identité francophone, le secrétaire général

1982 sous le titre Shrayber vos ikh hob gekent, memuarn un esayen [les écrivains que j’ai connus, mémoires et essais]. La traduction a été réalisée par l'auteur de cet article.

29. Voir à ce sujet les chapitres 5 et 6 dans Pierre Anctil, Le rendez-vous manqué, les Juifs de Montréal face au Québec de l'entre-deux-guerres (Québec, l’Institut québécois de recherche sur la culture, Montréal, 1988) et Pierre Anctil, "A. M. Klein: du poète et de ses rapports avec le Québec français ", Journal of Canadian Studies/Revue d'études canadiennes, 19,2 (1984): 114-131.

30. On ne trouve pas de preuves directes de ce fait dans les archives de H.-M. Caiserman. Il existe toutefois plusieurs allusions à cette volonté de dialogue, entre autres dans ce texte: Hananiah-Meir Caiserman, "A tip fun Kanader Frantsoyzen [un type de Canadien français]», Der Keneder Odler [1'Aigle canadien], 19 octobre 1936. Cet article laisse voir l'empathie que l'activiste ressentait pour le Canada français. 
du Congrès juif canadien se serait sans doute vite enlisé après 1934 dans un combat perdu d'avance, faute de posséder une vision juste de la société canadienne-française. Parmi les Juifs canadiens actifs dans la vie communautaire, Caiserman était sans doute le seul à cette époque qui était animé d'un désir de compréhension mutuelle et qui possédait les outils nécessaires à la mise en place d'une rencontre véritable entre les deux groupes. Il faut toutefois attendre le début des années 1950 pour que ces efforts portent fruit, et que le Congrès juif canadien s'engage résolument à établir un dialogue permanent entre Juifs et francophones.

Pour Caiserman, le Canada français avait constitué dès le départ une composante incontournable de la réalité canadienne, conviction qui avait été réaffirmée très tôt dans son esprit quand il s'était engagé sur les sentiers luxuriants de la poésie nationaliste d'expression française. Comment expliquer autrement son engouement pour un univers de signification littéraire apparu au milieu du XIX ${ }^{\mathrm{e}}$ siècle, et qui était porteur en filigrane d'un important projet d'affirmation culturelle. Cette découverte, le critique littéraire avait un temps pensé la transmettre à ses coreligionnaires, confrontés comme les Canadiens français à un statut de minoritaires au sein de leur nouveau pays. Certes les Juifs est-européens n'avaient pas participé aux premières tentatives de colonisation au pays, et ils avaient subi de surcroît une expérience de discrimination combien plus intense dans l'Ancien Monde.

Pour Caiserman cependant, il n'était pas inconcevable que l'attitude des francophones offre aux immigrants yiddishophones une clé d'interprétation de l'histoire canadienne. Tendre le regard vers le Canada français, c'était aussi lutter contre l'antisémitisme et le risque de nouvelles persécutions qu'une ignorance mutuelle ne faisait que raviver. Cette conviction était si forte chez Caiserman qu'il réussit à la transmettre à David Rome $^{31}$ lorsque le jeune Vancouvérois fit de Montréal son nouveau port d'attache. Comme Caiserman au départ, Rome ignorait tout du Canada français lorsqu'il devint responsable, en 1942, des relations publiques au Congrès juif canadien. Rome, comme son maître, parlait sans doute le français à grand peine à cette époque. Les circonstances de l'après-guerre allaient toutefois permettre à Rome d'aller beaucoup plus loin que son mentor décédé en décembre 1950, soit à un moment où l'Église catho-

31. Né en 1910 à Vilnius en Lituanie, David Rome était arrivé au Canada en 1921 et s'était immédiatement installé avec sa famille à Vancouver. Éduqué en anglais dans son pays d'adoption, il possédait aussi une très bonne connaissance du yiddish. 
lique commençait à peine à entrevoir l'importance d'amorcer un dialogue de fond avec le judaïsme.

La découverte du manuscrit de Caiserman sur la littérature canadiennefrançaise montre qu'il reste encore beaucoup de travail à accomplir pour mieux saisir les perceptions de la génération issue de la grande migration ainsi que les gestes que ces nouveaux venus ont faits au moment de leur arrivée au pays. La période initiale de 1905-1925 est encore peu connue des chercheurs parce que la plupart des documents qui s'y réfèrent sont écrits en yiddish et recouvrent en partie une expérience culturelle issue de l'élan révolutionnaire russe de 1905 et de 1917.

Ces premiers militants et activistes n'en jetèrent pas moins les bases des principales organisations communautaires juives de Montréal, en plus de définir des approches et des attitudes qui s’avéreront cruciales en regard des avancées ultérieures de l'histoire juive canadienne. Il y a aussi que les yiddishophones ont développé, face au Canada français, une compréhension qui différerait de manière notable de celle adoptée par la génération suivante des Juifs ashkénazes nés à Montréal, et éduqués pour la plupart dans le milieu scolaire anglo-protestant, c'est-à-dire sans contacts soutenus avec les réalisations culturelles de langue française. Plus cosmopolites et peu influencés par les conflits politiques ayant cours au Canada entre les deux communautés dominantes, les immigrants de langue yiddish avaient de surcroît vécu en Europe de l'Est de nombreuses situations linguistiques et culturelles qui n'étaient pas sans faire penser au pluralisme ayant cours à Montréal depuis le début du $\mathrm{XX}^{\mathrm{e}}$ siècle. Pour cette raison peut-être, plusieurs d'entre eux redécouvrirent au pied du mont Royal un contexte qui leur était familier depuis longtemps, et avec lequel ils se trouvaient relativement à l'aise, incluant une forte présence francophone à laquelle ils ne voulurent pas tourner le dos. 